\title{
Control of Director Fields in Phospholipid-Coated Liquid Crystal Droplets
}

Daniel A. Paterson, Peng Bao, Radwa H. Abou-Saleh, Sally A. Peyman, J. Cliff Jones,
Jonathan A. T. Sandoe, Stephen D. Evans, Helen F. Gleeson, and Richard J. Bushby*

Cite This: Langmuir 2020, 36, 6436-6446

Read Online

ACCESS

Wll Metrics \& More

Article Recommendations

Supporting Information

ABSTRACT: In liquid crystal (LC) droplets, small changes in surface anchoring energy can produce large changes in the director field which result in readily detectable optical effects. This makes them attractive for use as biosensors. Coating LC droplets with a phospholipid monolayer provides a bridge between the hydrophobic world of LCs and the water-based world of biology and makes it possible to incorporate naturally occurring biosensor systems. However, phospholipids promote strong perpendicular (homeotropic) anchoring that can inhibit switching of the director field. We show that the tendency for phospholipid layers to promote perpendicular anchoring can be suppressed by using

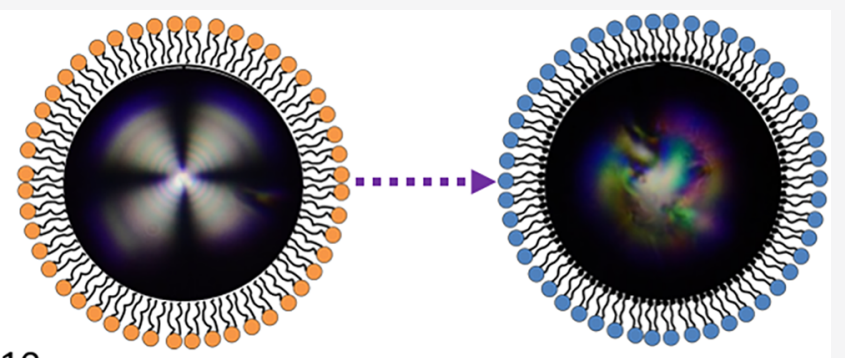
synthetic phospholipids in which the acyl chains are terminated with bulky tert-butyl or ferrocenyl groups; the larger these end-group(s), the less likely the system is to be perpendicular/radial. Additionally, the droplet director field is found to be dependent on the nature of the LC, particularly its intrinsic surface properties, but not (apparently) on the sign of the dielectric anisotropy, the proximity to the melting/isotropic phase transition, the surface tension (in air), or the values of the Frank elastic constants.

\section{INTRODUCTION}

It has long been known that liquid crystals (LCs) are present in a large number of biological systems, but despite this, researchers have only relatively recently realized that the effects of biological stimuli on LCs, in particular the way that they affect LC alignment, could be used in devices. This rich new vein of exploration has already demonstrated the potential of LC systems as biosensors. ${ }^{1-6}$ Biosensors incorporating a LCaqueous interface have been shown for both LC films ${ }^{7-9}$ and LC droplets, ${ }^{10-14}$ and they have been used for the successful detection of antigens, pathogens, lipids, cytotoxins, proteins, ${ }^{15}$ surfactants, and, more recently, antimicrobial peptides. ${ }^{16}$ In the majority of cases, the LC systems used have been calamitic nematic, often 4 -cyano- $4^{\prime}$-pentylbiphenyl (5CB) or E7, ${ }^{16}$ both of which are relatively simple cyanobiphenyl-based systems. Most sensors rely on a switch of anchoring that influences the organization of the bulk director field, resulting in a detectable change in optical properties. The principal challenge in designing LC droplet systems for use as biosensors is the need for both high sensitivity and specificity. The latter requires tailoring of the $\mathrm{LC} /$ water interface so that it responds to specific biological interactions. In this respect phospholipidcoated droplets are particularly attractive since they hold the prospect of being able to exploit some of the specific analyte recognition systems found in natural biomembranes. However, one potential limitation is the well-known tendency of phospholipid coatings to promote strong perpendicular (homeotropic) anchoring, something that can make switching of the director field very difficult. Past studies have demonstrated that for LC droplets the anchoring of the LC is dependent on many factors. These include the structure and density of the surfactant coating, the interfacial anchoring energy, ${ }^{4}$ a size-dependent effect relating to the elasticity of the LC, ${ }^{17}$ and the strength of interactions between LC molecules in the bulk. ${ }^{18}$ Experiments have shown that the elastic energy, $F_{\mathrm{E}}$, scales linearly as $F_{\mathrm{E}} \sim K R$ for a droplet of radius $R$, whereas the surface energy $F_{s}$ scales as $F_{s} \sim W R^{2}$ where $W$ is an anchoring energy coefficient that controls the orientation of LC molecules at a surface. From this a critical length can be determined $R \sim K / W$, above which the surface anchoring energy dominates or below which the elastic energy dominates. $^{19}$

For a typical LC droplet in water sized $1-100 \mu \mathrm{m}$ it has been shown that surfactant or lipid adsorption to the surface can induce changes in the alignment of the LC from bipolar to

Received: March 6, 2020

Revised: May 11, 2020

Published: May 11, 2020 
radial (Figure 1). Indeed, our own work has demonstrated that the reverse of this effect can be used to cause an optical
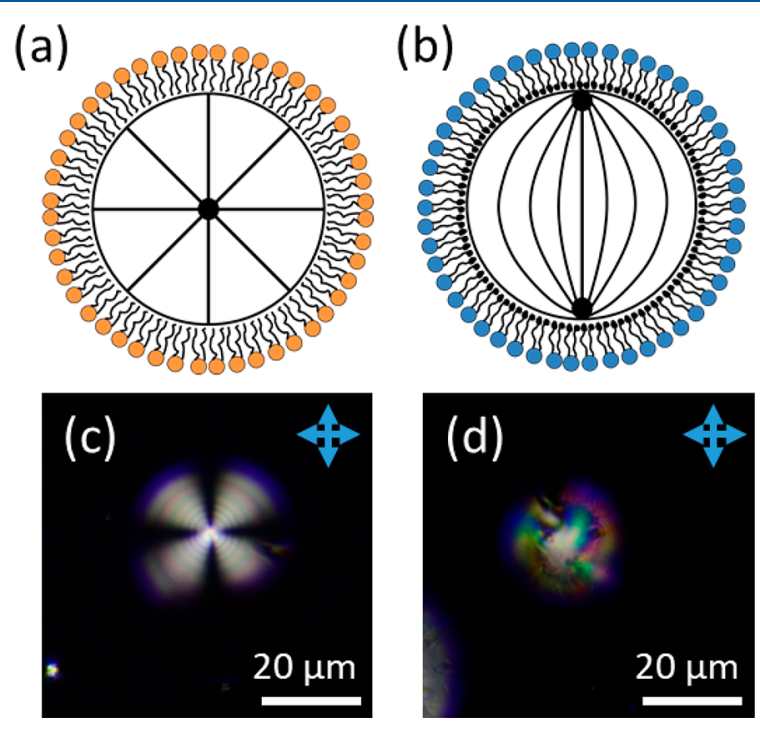

Figure 1. Schematic drawing and POM images of the two most common director fields seen in LC droplets. (a, c) Radial director field (promoted by most lipid coatings) and (b, d) bipolar director field (which we show are promoted by modified lipids in which the acyl chains are terminated with bulky end groups). In the case of a radial director field, the $\mathrm{LC}$ director is perpendicular to the surface and there is a defect at the center of the droplet. In the bipolar case, the director field of the LC is oriented planar (parallel to the interface) with two surface defects (boojums) at the poles.

response associated with the switching of radial to bipolar droplets when the lipid coating is removed from the LC through interaction with the toxin SMP43. ${ }^{16}$ In real terms there are several droplet configurations, some of which are stable, that are intermediate to radial and bipolar alignment: radial-preradial/escaped radial (where the defect point lies near or beyond the droplet surface)-axial (exhibiting an equatorial disclination loop)-bipolar in which the surface alignment changes from perpendicular to tilted to planar. $^{20,21}$

For surfactant-coated LC droplets and films, there have been several studies of the role of LC/surfactant interactions in determining the surface orientation of the director. ${ }^{22-24}$ For a typical linear (unbranched) alkyl chain surfactant of sufficient surface density, a perpendicular (homeotropic) director field is observed. However, in the case of branched chain surfactants, a planar alignment is observed. This has been rationalized in terms of the ability of branching of the chain to suppress interdigitation between the LC molecules and surfactant monolayer. $^{23}$

In this paper we address a limitation posed by a phospholipid coating — that of (normally) strong perpendicular (homeotropic) anchoring of the LC at the LC/phospholipid interface. We describe the synthesis of a series PC lipids in which the acyl chains are terminated with bulky substituents (Figure 2) which inhibit interdigitation and hence suppress perpendicular anchoring. tert-Butyl and the larger ferrocenyl group were chosen as the two acyl end-chain substituents. The pure compounds $5 \mathrm{CB}$ and $6 \mathrm{CB}$ and mixtures E7, MLC7023, MLC2018, and MLC6609 were used as the nematic LCs, giving a range of differing LC types and physical properties. The results (summarized in Table 1) show the expected

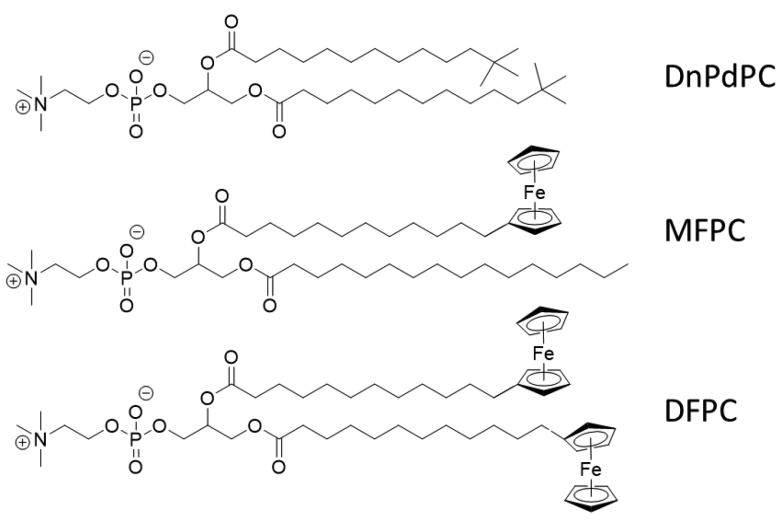

Figure 2. Molecular structures of the synthesized lipids: 1,2-di-neopentadecoyl-sn-glycero-3-phosphocholine (DnPdPC), 1-palmitoyl-2(12'-ferrocenyl)-dodecoyl-sn-glycero-3-phosphocholine (MFPC), and 1,2-bis(12'-ferrocenyl)-dodecanoyl-sn-glycero-3-phosphocholine (DFPC).

dependence of LC alignment on the ease of interdigitation, but they also show that the LC material is critical. Investigation of the dependence of the director field on the nature of the LC shows that (at least for these droplets) it is the intrinsic surface rather than the bulk properties of the LC that matter most.

\section{EXPERIMENTAL PROCEDURES}

Materials. The liquid crystals 4-cyano-4'-pentylbiphenyl (5CB), 4cyano-4'-hexylbiphenyl (6CB), and the mixture E7 were purchased from Synthon Chemicals GmbH \& Co. KG, Germany. The mixtures MLC7023, MLC2081, and MLC6609 were provided by Merck Group, UK. The premium glass microscope slides were from Fisher Scientific (Pittsburgh, PA). 1,2-Dioleoyl-sn-glycero-3-phosphocholine (DOPC) and 1,2-dioleoyl-sn-glycero-3-phospho-rac-(1-glycerol) sodium salt (DOPG) were purchased from Sigma-Aldrich. All aqueous solutions were prepared by using deionized water from a Milli-Q water purification system (Millipore, Bedford, MA).

Synthesis and Characterization of the Lipids. Details of the synthesis of the lipids DnPdPC, MFPC, and DFPC and related spectroscopic data are provided in the Supporting Information.

Formation of Lipid-Coated Liquid Crystal Droplets. To formulate our LC droplets, a liposome solution was made by hydration and tip sonication of a dried lipid or mixture of lipids with $0.1 \mathrm{~mol} \%$ Texas Red-DHPE, as described previously. ${ }^{25}$ Texas RedDHPE is included to allow fluorescence imaging of the lipid-coated droplets, thereby allowing confirmation of the presence (or absence) of the lipid on the droplet. The lipid mixture was dissolved in a chloroform/methanol 1:1 mixture in a glass vial and dried under nitrogen flow for $1 \mathrm{~h}$. Milli- $\mathrm{QH}_{2} \mathrm{O}$ was then added, and the vial was vortex-mixed for $2 \mathrm{~min}$ to produce a suspension of the lipids. The suspension was tip-sonicated for $30 \mathrm{~min}$ at $4{ }^{\circ} \mathrm{C}$ until a clear solution could be observed and then centrifuged for $5 \mathrm{~min}$ at $5000 \mathrm{rpm}$. The resultant supernatant was decanted to remove metal sediment from the tip sonicator. The supernatant was diluted with $\mathrm{H}_{2} \mathrm{O}$ to give a concentration of lipid of $0.5 \mathrm{mg} / \mathrm{mL}$. The liposome solution was stored at $4{ }^{\circ} \mathrm{C}$. In all cases the LC droplets were formed by using mechanical methods, as reported elsewhere, ${ }^{26}$ usually by using a combination of vortexing and agitation by hand. $2 \mu \mathrm{L}$ of LC in in 100 $\mu \mathrm{L}$ of continuous aqueous solution of lipids and was enough to lead to a sufficiently dilute emulsion of LC droplets with minimal coalescence and a variety of droplet sizes.

Optical Studies of the Lipid-Coated Liquid Crystal Droplets. The LC droplets were suspended in aqueous solution and held between a glass microscope slide and a glass coverslip separated by an $\sim 400 \mu$ m deep polydimethylsiloxane (PDMS) well. To make the well, a PDMS thin film was formed by pouring $2 \mathrm{~g}$ of fully mixed elastomer and curing agent (weight ratio 10:1) (Dow Corning Sylgard 184 kit) 
Table 1. Director Fields Observed for LC Droplets Formed from Different LC Materials, Coated with Phospholipid Mixtures (All Observations Are at Room Temperature)

\begin{tabular}{|c|c|c|c|c|}
\hline material & DOPC:DOPG & DnPdPC & MFPC & DFPC \\
\hline MLC6609 & radial & radial & radial & radial \\
\hline E7 & radial & radial & radial & radial \\
\hline $6 \mathrm{CB}$ & radial & radial & radial & nonradial \\
\hline $5 \mathrm{CB}$ & radial & radial & $\operatorname{radial}^{a}$ & nonradial \\
\hline MLC2081 & radial & nonradial $^{b}$ & nonradial & nonradial $^{c}$ \\
\hline MLC7023 & nonradial & nonradial & nonradial & nonradial \\
\hline
\end{tabular}

${ }^{a}$ For these droplets both radial and preradial droplets are observed, but it was noted that droplets were primarily radial. ${ }^{b}$ For these droplets a mixture of droplet alignments was observed (including preradial and escaped radial), but it was noted that droplets below $15 \mu \mathrm{m}$ were primarily bipolar in this case. ${ }^{c}$ For these droplets both axial and bipolar droplets are observed, but it was noted that droplets were primarily bipolar.

into a plastic Petri dish (100 $\mathrm{mm}$ in diameter) and leaving at room temperature for $48 \mathrm{~h}$ on a horizontal surface. Then, a piece of PDMS thin film $\left(1.5 \times 1.5 \mathrm{~cm}^{2}\right)$ was cut from the PDMS film in the Petri dish. A hole of $9 \mathrm{~mm}$ in diameter was made by using a homemade punch. The PDMS sheet was placed on the microscope slide. The LC droplet sample was deposited into the middle of the hole using a pipet, and the top was sealed with a coverslip $(0.12 \mathrm{~mm}$ thick $)$. Phase characterization was performed by using a DM 2700 M Leica Microsystems Ltd. polarized light microscope equipped with a pair of linear polarizers and a Nikon D3000 camera, combined with a Linkam T95 Peltier hot stage. The fluorescence signal from the Texas RedDHPE fluorophores included in the lipid layers was observed by using an epifluorescence microscope (Nikon Instruments Europe B. V., Kingston, UK) equipped with a Texas Red filter block. Fluorescence images were captured by using either an Andor Zyla sCMOS camera (Oxford Instruments plc, UK) or an Orca-ER (Hamamatsu).

Langmuir Trough Experiments on the Lipids. A Langmuir trough (KSV Nima) was used as previously described ${ }^{27}$ to determine the area per molecule, collapse pressure, and monolayer compressibility. ${ }^{28}$ The trough was equipped with two movable PTFE barriers to compress the monolayer symmetrically. A Wilhelmy plate tensiometer (paper method) was used to measure the surface pressure of the monolayer. For this experiment, $25 \mu \mathrm{L}$ of a $1 \mathrm{mg} / \mathrm{mL}$ solution of each lipid in chloroform was spread on the surface of the ultrapure water subphase and allowed $10 \mathrm{~min}$ to ensure the evaporation of chloroform before starting the compression. The barrier compression was performed at a constant speed of $5 \mathrm{~mm} / \mathrm{min}$. The isotherms were collected for four repeats per lipid to confirm reproducibility with the surface pressure not allowed to increase beyond $35 \mathrm{mN} / \mathrm{m}$ for the first three trials, but for the last trial it was allowed to reach the collapse pressure. The area per lipid molecule is an average of three trials in each case excluding the first one.

Differential Scanning Calorimetry (DSC) of the Lipids. We investigated the thermal behavior of the new lipids by DSC using a TA Instruments Q20 differential scanning calorimeter equipped with an AQ20 autosampler and a RCS90 refrigerated cooling system, calibrated by use of indium standards. The sample was prepared by using excess water (comparative runs with both a 20 -fold and a 40 fold molar excess) and a heat-cool-reheat cycle performed at $10{ }^{\circ} \mathrm{C}$ $\mathrm{min}^{-1}$ with a $3 \mathrm{~min}$ isotherm between heating and cooling segments. Thermal data were extracted from the reheat trace. Experiments on hydrated DOPC gave values for the $\mathrm{L}_{\alpha} \mathrm{L}_{\beta}$ transition temperature and enthalpy in good agreement with the literature values.

Fluorescence Recovery After Photobleaching (FRAP) of the Lipids. The fluorescence recovery after photobleaching (FRAP) experiments was performed by using an epifluorescence microscope (E600 Nikon, USA). A high-pressure mercury arc lamp was used for bleaching the lipid bilayers. The bleached spots were $28 \mu \mathrm{m}$ in diameter, and the bleaching time was $30 \mathrm{~s}$. A series of time-lapse fluorescence images after photobleaching were collected by using a Zyla sCMOS CCD camera (Andor Technology Ltd., Belfast, UK).
The diffusion coefficient of the fluorescent Texas Red-DHPE lipid additive was calculated by following the Axelrod method. ${ }^{29}$

Measurement of the Elastic Constants of the Liquid Crystals. To measure the splay $\left(K_{11}\right)$ and bend $\left(K_{33}\right)$ elastic constants, a technique previously reported by Srigengan and Kaur was employed $^{30,31}$ using analysis of the electric-field-induced Freedericksz transition in appropriate geometries. All dielectric measurements were performed using devices of nominal thickness $5 \mu \mathrm{m}$, supplied by AWAT. Homeotropic alignment was achieved by using the polyimide SE1211, while planar alignment was obtained by using a rubbed (SE130) polyimide layer. The temperature of the devices was controlled with a relative accuracy of $\pm 0.1^{\circ} \mathrm{C}$ by using a Linkam LTS 350 hot stage and a TMS 94 temperature controller. All electric fields were applied at a frequency of $2 \mathrm{kHz}$, avoiding any relaxations in the systems. All devices were capillary filled in the nematic phase. The surface alignment on the devices provided excellent monodomains, and the well-defined sample geometries allowed experiments to be performed that determined the dielectric and the splay and bend elastic constants for the wide variety of LC materials considered with an accuracy of $5 \%$.

Measurement of the Surface Tension of the Liquid Crystals. The pendant drop (or hanging drop) technique ${ }^{32}$ was utilized to measure the surface tension of the LCs. The data were taken by using a FTÅ4000 instrument from First Ten Ångstroms, USA. The needle tips used were either 21 or 22 gauge PTFE-coated needles supplied by Nordson, UK. A drop was formed on the tip of the PTFE-coated needle via syringe. The drop was illuminated by a diffuse light source and imaged by a high-speed camera. An image was collected, and the contour of the drop (as it grew) was fitted to the Young-Laplace equation by using a software plugin for ImageJ. ${ }^{33}$ The software provided the surface tension and drop volume. The instrument was calibrated to the diameter of the needle. Because the experiment is very sensitive to small vibrations or changes in the air pressure (which cause oscillation of the drop and, in turn, the contour used in the Young-Laplace equation), measurements were taken by using steps to minimize disruptive air flow, and the droplet was given time to equilibrate on the tip of the needle before images were taken. A large number of runs were undertaken to calculate the surface tension including measuring various drop sizes. As shown in the example in Figure S35 (the data for 5CB), the "apparent (computed) surface tension" is a function of the droplet volume. This increases up to a volume of ca. $4 \mu \mathrm{L}$. The "true" surface tension is reached at the plateau defined by a plot of this kind at a volume of ca. $5 \mu \mathrm{L}$. In all cases the reported values of the surface tension are taken from this plateau region and are averaged over several experiments. Above $5 \mu \mathrm{L}$ the needle is no longer able to support the droplet.

Alignment of the Liquid Crystals at the Air Interface and on OTS-Coated Slides. To check the alignment of LCs at a free air interface, a hole $\approx 1.5 \mathrm{~mm}$ in diameter was drilled into a glass slide with a Dremel diamond bit. The glass slide was subsequently cleaned by sonication with methanol and dried in an oven at $100{ }^{\circ} \mathrm{C}$ for $2 \mathrm{~h}$. 

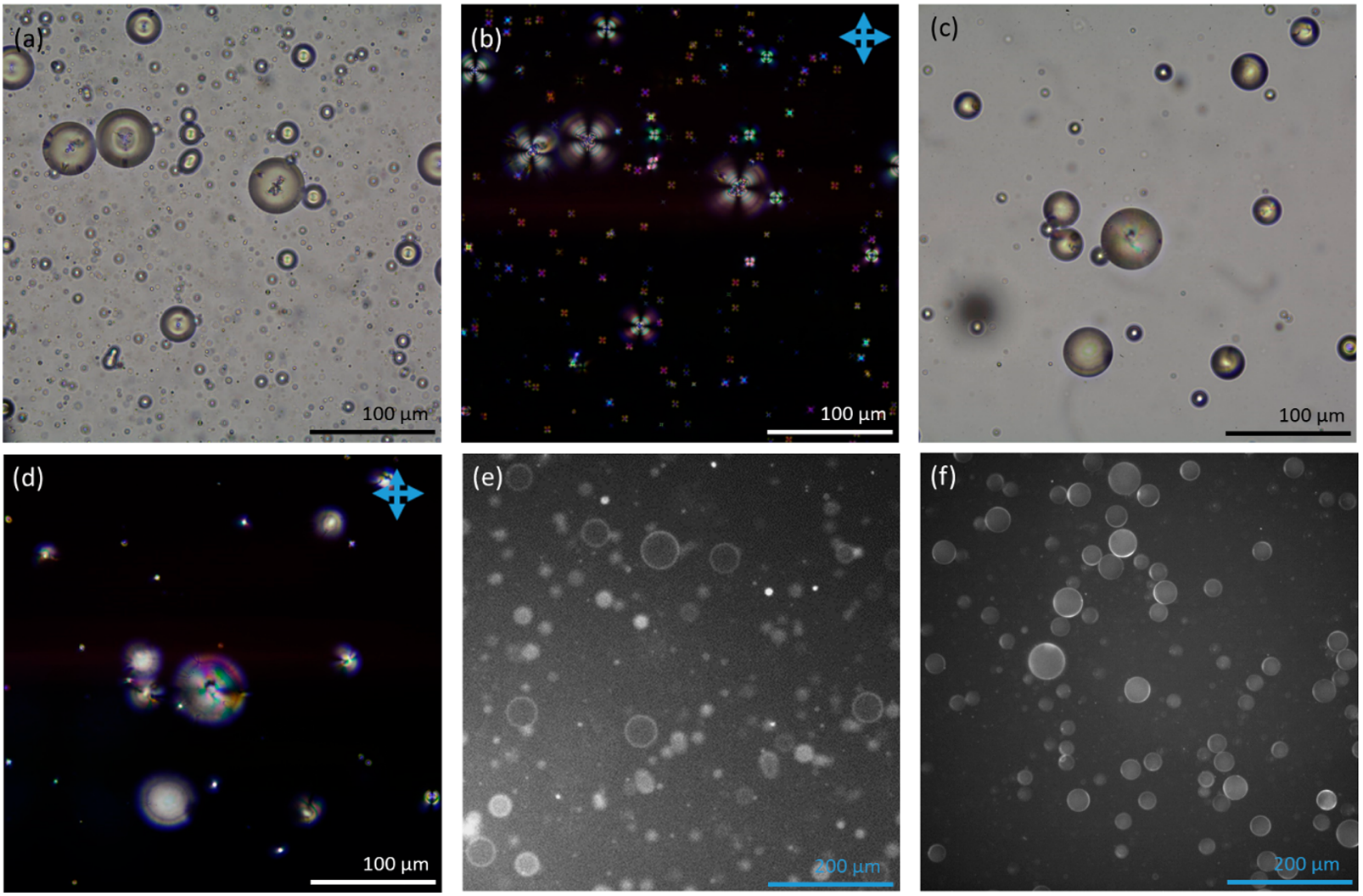

Figure 3. Transmission images of droplets of 6CB: (a) bright-field image of 6CB coated in DnPdPC; (b) image taken between crossed polarizers of 6CB coated in DnPdPC; (c) bright-field image of 6CB coated in DFPC; $(d)$ image taken between crossed polarizers of 6CB coated in DFPC; (e) a fluorescence image of DnPdPC-coated 6CB droplet before excess liposome has been removed from solution; (f) a fluorescence image of DFPCcoated $6 \mathrm{CB}$ droplet once excess liposome has been removed from solution. All measurements were made at room temperature.

To deposit LC within the cavity, $5 \mu \mathrm{L}$ of LC was pipetted onto the glass slide near the cavity, and a film was created by smearing the LC across the hole by using the flat edge of a razor blade.

The alignment of LCs at an octadecyltrichlorosilane (OTS)-coated glass interface was determined by sonicating glass slides in a Decon 90 and deionized water solution at $60{ }^{\circ} \mathrm{C}$ for $1 \mathrm{~h}$ before rinsing with water, methanol, and IPA before drying in an oven at $100{ }^{\circ} \mathrm{C}$ for $2 \mathrm{~h}$ prior to OTS deposition. A $10 \mathrm{mM}$ OTS solution was prepared by adding OTS to hexane, and the glass slides were then immersed in this solution for $1 \mathrm{~h}$ at $25^{\circ} \mathrm{C}$. They were then rinsed by sonicating in hexane for $15 \mathrm{~min}$ followed by washing with dichloromethane, IPA, and lastly water. The slides were then dried in an oven at $100{ }^{\circ} \mathrm{C}$ for 2 $\mathrm{h}$ prior to use. The quality of the OTS treatment was then checked by sandwiching two slides to form a LC cell. The LC was then introduced, and the alignment was observed via polarized optical microscopy to confirm homeotropic alignment. The slides were then used for each of the LCs of interest. In each case the LC was heated into the isotropic phase and slowly cooled into the nematic phase to produce well-aligned samples. The OTS used for functionalizing the glass slides for the LC alignment studies was obtained from SigmaAldrich.

\section{RESULTS AND DISCUSSION}

Characterization of the Lipid-Coated Liquid Crystal Droplets. The lipid-coated LC droplets were produced by shaking the LC in an aqueous solution containing phospholipid liposomes rather than by the microfluidic method which we used previously. ${ }^{16}$ In the present case, examining a range of droplet sizes allowed us to determine whether the results were size-dependent. Alignment was assigned on the basis of POM observation for freely suspended droplets in solution. In the case of a radial director field (perpendicular alignment of the director at the surface) a typical Maltese cross was observed in the crossed polarizer images (Figures $1 \mathrm{c}$ and $3 \mathrm{~b}$ ). In bright-field images very small droplets can be observed within the LC droplets. This is a phenomenon previously reported ${ }^{34-36}$ (Figure 3a). For droplets with a bipolar director field (planar alignment) a more complex image is observed (Figures $1 \mathrm{~d}$ and $3 \mathrm{c}$ ). For bipolar alignment with two boojums located at opposite poles, the optical texture differs depending on the orientation of these boojums relative to the observer. For droplets exhibiting preradial/escaped radial director fields which have a tilted surface alignment, a distorted Maltese cross with a defect point off center of the droplet is observed (Figure S21e,f). For droplets with an axial director field (which also indicates a tilted surface alignment), an equatorial disclination line can be observed in the bright-field image (Figure S25g,h). Images for all other LC/lipid droplet systems studied are included in Figures S21-S26. Experimentally, the clearest observation to make is in defining which systems exhibit radial alignment and which exhibit a nonradial alignment.

We were able to confirm the presence of a complete lipid monolayer on the LC droplets using fluorescence microscopy, taking advantage of the low percentage of fluorescently labeled lipid (Texas Red-DHPE) in the lipid mixture (Figure 3e,f). For this experiment, it was important to resuspend the droplets in liposome-free water. Droplets in a solution still containing excess liposomes show a very bright fluorescent ring around each droplet which is due mainly to lensing of the background solution fluorescence from excess liposomes. Only after resuspension in liposome-free solution (Figure 3f) is the 
background nonfluorescent so that the observed fluorescent ring can be confidently assigned to the lipid monolayer. In each case this fluorescent ring was a continuous halo around the droplet, but in some cases it appears to be nonuniform. This nonuniformity is due to the proximity of neighboring droplets or to droplets in other planes of the sample causing lensing effects. The lipid concentration used in this study should produce a monolayer with full coverage over the surface of the LC droplet, and this has been confirmed by comparison with the fluorescence signal of supported bilayers previously reported. ${ }^{37}$ We see no apparent partition of lipids around defect centers in the LC droplets, as is observed by Abbott et al. $^{38,39}$

The droplet director fields observed for each LC-lipid combination are summarized in Table 1 . In all but one case, the alignment observed was the same for all droplet sizes between ca. 1 and ca. $100 \mu \mathrm{m}$. The exception was DnPdPCcoated MLC2081, where the smaller droplets exhibited a bipolar director field but the largest ones (ca. 15-100 $\mu \mathrm{m}$ ) showed other nonradial director fields. What is immediately obvious from Table 1 is that the observed director field depends on both the nature of the lipid monolayer and that of the LC. A detailed characterization of the properties of the LC and phospholipid components (described below) was undertaken in an attempt to understand this dependence, and it is these studies that provide the justification for the order in which the data in Table 1 have been arranged.

Characterization of the Novel Phospholipids. In Table 1 , the lipids have been ordered on the basis of "ease of LC/ phospholipid interdigitation". The estimate of the size of the acyl chain end-groups (DOPC:DOPG being the smallest and DFPC the largest) is based on measured molecular volumes and molecular areas ${ }^{40-42}$ and on space-filling molecular models. However, to confirm these assumptions, we measured the cross-sectional areas for these lipids at the air-water interface using the Langmuir trough method. The results of these experiments are displayed in Figure 4 and summarized in Table 2.

The measured value for the surface area per molecule (extrapolated to zero surface pressure) for the "standard" lipid DPPC in its liquid condensed phase $\left(0.49 \pm 0.02 \mathrm{~nm}^{2}\right)$ is in agreement with data published elsewhere. ${ }^{\overline{43}}$ For the other lipids the areas follow the expected order (DFPC > MFPC > DnPdPC). For PC lipids it is known that the area per lipid molecule in monolayers and in bilayers (the conformation and hence the area occupied by the headgroup) can vary to accommodate the nature and order of the acyl substituents although areas are usually in the region $0.5-0.9 \mathrm{~nm}^{2}$. In this case the effect of the end-chain substituents on the surface area per molecule is reasonable for a normal "chain-extended" conformation of the lipid molecules (given the known values for the group volumes and group cross-sectional areas ${ }^{40-42}$ ). In each case the values obtained (Table 2 and Figure $4 \mathrm{~b}, \mathrm{c}$ ) confirm that the "areas per molecule" are associated with the liquid-condensed (chain-ordered) state and are consistent with similar systems previously reported. ${ }^{44}$ The areas per molecule (particularly that for DFPC) would be difficult to reconcile with a model in which the ferrocenes are located close to the water. Previous work on "bolaform" amphiphiles of the $\mathrm{Me}_{3} \mathrm{~N}^{+}\left(\mathrm{CH}_{2}\right)_{n}[\mathrm{Fe}]$ type have established that for shorter chain homologues both the trimethylammonium and the ferrocene lie close to the surface of the water, but for longer chains an extended conformation is observed in which only the (a)

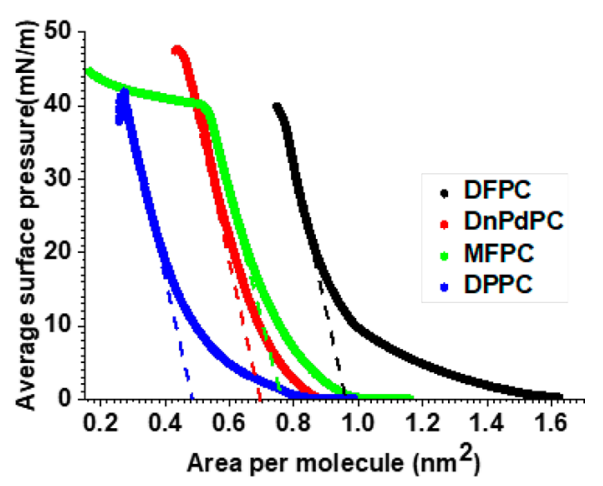

(b)

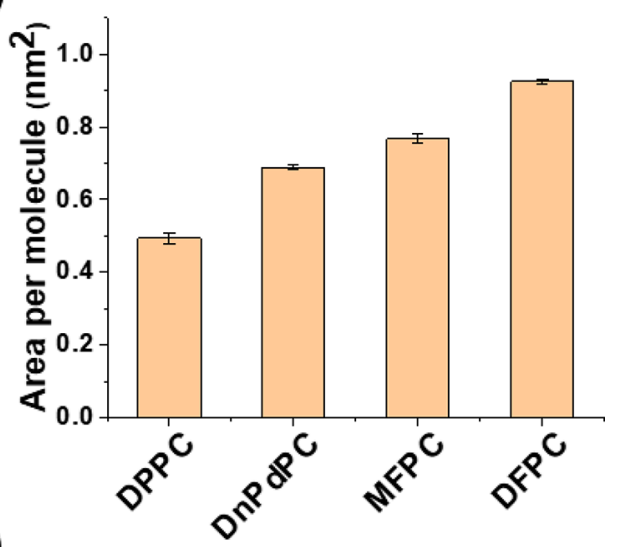

(c)

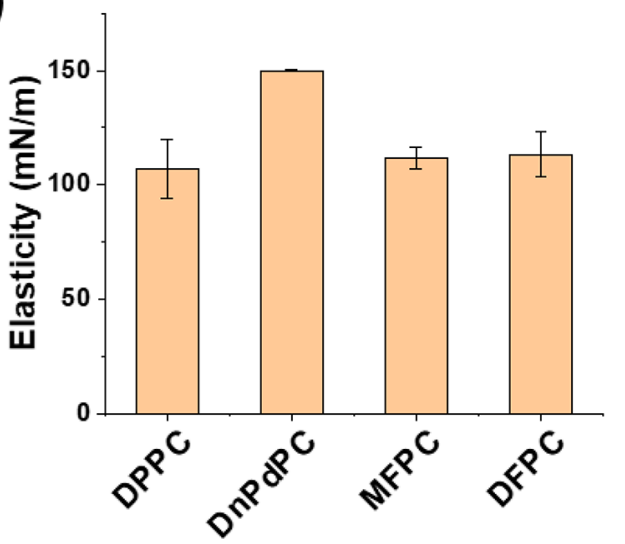

Figure 4. Langmuir trough data for the phospholipid monolayers on water. (a) Molecular area versus surface pressure. (b) Limiting molecular surface area per molecule (extrapolated from (a)). (c) Elasticity values.

Table 2. Summary of Lipid Properties: Data for the Hydrated Bilayers from DSC and for the Monolayer at the Air/Water Interface from Langmuir Trough Experiments

$\begin{array}{llccc}\text { material } & \begin{array}{c}T_{\mathrm{m}} \\ \left({ }^{\circ} \mathrm{C}\right)\end{array} & \begin{array}{c}\Delta H \\ \left(\mathrm{~J} \mathrm{~g}^{-1}\right)\end{array} & \begin{array}{c}\text { surface area } \\ \left(\mathrm{nm}^{2}\right)\end{array} & \begin{array}{c}\text { elasticity } \\ \left(\mathrm{mN} \mathrm{m}^{-1}\right)\end{array} \\ \text { DPPC } & & & 0.49 \pm 0.02 & 107 \pm 12.7 \\ \text { DnPdPC } & <0 & & 0.69 \pm 0.02 & 150 \pm 0.3 \\ \text { MFPC } & 25.1 & 15.5 & 0.77 \pm 0.02 & 112 \pm 4.8 \\ \text { DFPC } & 11.5 & 29.7 & 0.96 \pm 0.02 & 113 \pm 10.0\end{array}$

trimethylammonium is close to the water surface. ${ }^{45}$ For such amphiphiles their ability to induce planar anchoring of LCs has been attributed to this particular conformation of the amphiphile ${ }^{22,46}$ rather than to inhibition of interdigitation. ${ }^{23}$ However, for example, in the case of DFPC such an 
arrangement should lead to a surface area per molecule of the order of ca. $1.7 \mathrm{~nm}^{2}$ (of the order of twice that of $\left.\mathrm{Me}_{3} \mathrm{~N}^{+}\left(\mathrm{CH}_{2}\right)_{11}[\mathrm{Fe}]\right){ }^{45}$

The argument for interdigitation of the LCs into the lipid tail region as being the driving force for perpendicular alignment of the director field requires that the acyl chains of the phospholipid monolayers are in a fluid/disordered state. For monolayers, direct evidence for this is difficult to obtain. However, some support for the idea comes from studies of the corresponding bilayers by differential scanning calorimetry (DSC) and from FRAP studies (fluorescence recovery studies of the hydrated bilayers supported on glass).

DSC experiments were performed on fully hydrated samples of the lipids in which liposomes (or other highly hydrated bilayer phases) are expected to be present. DnPdPC showed no transitions between 0 and $80{ }^{\circ} \mathrm{C}$. The chain-melting transition lies outside of this temperature range. On heating, the DSC for hydrated DFPC showed a single endotherm at $25.1{ }^{\circ} \mathrm{C}\left(15.5 \mathrm{~J} \mathrm{~g}^{-1}\right)$. MFPC showed a broader endotherm (with some evidence of a shoulder; see Figures S18 and S19) at $11.5{ }^{\circ} \mathrm{C}\left(29.7 \mathrm{~J} \mathrm{~g}^{-1}\right)$. For both of these compounds, the enthalpy values observed are comparable to values of a chainmelting $\left(\mathrm{L}_{\beta}\right.$ or $\mathrm{P}_{\beta}{ }^{\prime}$ to $\left.\mathrm{L}_{\alpha}\right)$ transition in a hydrated $\mathrm{PC}$ bilayer. The results suggest that for hydrated bilayers of DFPC the transition from the $\mathrm{L}_{\beta}$ or $\mathrm{P}_{\beta^{\prime}}$ to the disordered $\mathrm{L}_{\alpha}$ phase lies just below and for MFPC just above room temperature.

We further characterized these lipids using FRAP of lipid bilayers supported on glass. For MFPC and DFPC this was not possible (stable supported bilayers could not be obtained). However, a supported bilayer of DnPdPC (containing a small percentage of Texas Red lipid) was made, and following photobleaching (Figure 5a), its fluorescence recovered over a
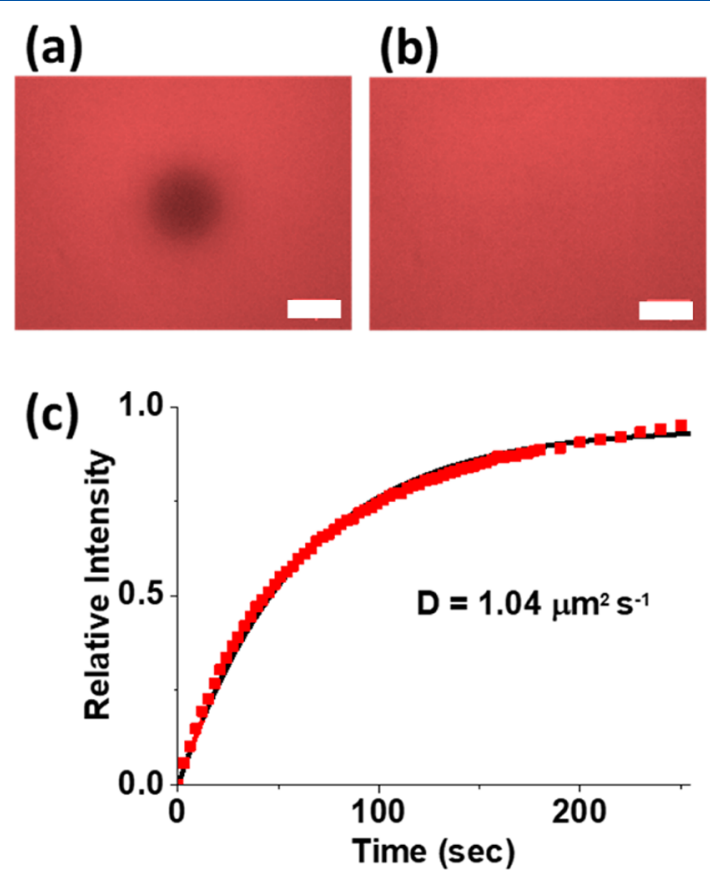

Figure 5. FRAP experiment for the supported lipid bilayer of DnPdPC with $0.1 \mathrm{~mol} \%$ Texas Red-DHPE at the glass/aqueous interface. (a) Fluorescence image of the lipid monolayer taken at immediately after photobleaching and (b) after recovery. (c) Recovery of the fluorescence signal in the bleaching region as a function of time (data points in red) and the theoretical fit (in black). ${ }^{29}$ Scale bar represents $20 \mu \mathrm{m}$. period of about $5 \mathrm{~min}$ as shown in Figure $5 \mathrm{~b}$. The diffusion coefficient measured, $1.04 \mu \mathrm{m}^{2} \mathrm{~s}^{-1}$, is comparable to that for other fluid supported PC lipid bilayers. ${ }^{25}$ This demonstrates that $\mathrm{DnPdPC}$ gives a fluid $\left(\mathrm{L}_{\alpha}\right)$ bilayer at room temperature. In combination with the DSC data, the FRAP studies suggest that in the bilayer at room temperature DnPdPC and DFPC, like DOPC/DOPG, are probably fluid but that for MFPC the fluid phase is formed just above room temperature.

Characterization of the Liquid Crystals. The studies of the phospholipids used for coating the droplets, which is described in the section above, show that the cross-sectional area for the monolayers (and hence the probable ease of interdigitation) follows the expected order (Figure 4 and Table 2 ). These studies also provide some support for the idea that the acyl chains are probably extended and in a fluid/disordered state. They help to justify the way the columns are arranged (how the "phase space" has been defined) ${ }^{47}$ in Table 1 (so far as the lipid component is concerned). The order within Table 1 in terms of the LC components (rows) is justified by the LC characterization studies below. We have used a wide range of different nematic LC systems, and their physical properties are summarized in Table 3 (and, for ease of comparison, the LCs have been placed in the same order as in Table 1).

Often the behavior of nematic LCs relates to the "reduced temperature" $T / T_{\mathrm{NI}}$ since this represents temperatures of similar order parameter $S$, given by Maier-Saupe theory. However, as can be seen from Table 3, there is no correlation between the observed droplet director fields at room temperature and the value of $T_{\mathrm{NI}}$.

A further possibility is that the surface alignment is influenced by a Coulombic interaction between the LC molecules and either the quadrupole of the ferrocenyl residues or the electric double layer associated with the phospholipid headgroup/water interface. Hence, we examined nematic LCs with both positive and negative dielectric anisotropy. In the former the molecular dipole is normally aligned more or less with the director, but in the latter it is normally more or less perpendicular to the director. As can be seen by comparing Tables 1 and 3, there is no correlation between the observed droplet director fields and either the sign or magnitude of the dielectric anisotropy.

In the literature, the behavior of director fields in LC droplets has often been discussed in terms of the bulk Frank elastic constants or in terms of their ratios. ${ }^{48}$ The simplest form of the radial director field only involves a splay deformation whereas that of the bipolar field involves a mixture of splay and bend components. Where these values were not available in the literature for the LCs we have used, we have measured them via the electric-field induced Freedericksz transition. These measured values for the elastic constants are similar to those for many simple nematic systems. In all cases $K_{11}$ is lower than $K_{33}$, and there is little variance in the ratio of $K_{11} / K_{33}$. As can be seen by comparing Tables 1 and 3 , there is no simple correlation between the observed droplet director fields and either $K_{11}$ (the splay elastic constant) or $K_{33}$ (the bend elastic constant) or the ratio between $K_{33}$ and $K_{11}$. Indeed, none of the bulk LC properties listed in Table 3 correlate with the director fields observed for the LC droplets (Table 1). We therefore turned our attention to a comparison of their intrinsic surface properties.

To study the behavior of the LCs at the air interface, a film of the LC was made over a $1.5 \mathrm{~mm}$ hole in a $1 \mathrm{~mm}$ thick glass slide. The presence of the LC film was easily confirmed by 
Table 3. Comparison of Physical Properties of the $\mathrm{LCs}^{a}$

\begin{tabular}{|c|c|c|c|c|c|c|c|c|}
\hline material & $\begin{array}{l}T_{\mathrm{NI}} \\
\left({ }^{\circ} \mathrm{C}\right)\end{array}$ & $\begin{array}{c}K_{11} \\
(\mathrm{pN})\end{array}$ & $\begin{array}{c}K_{33} \\
(\mathrm{pN})\end{array}$ & $K_{33} / K_{11}$ & $\Delta \varepsilon$ & $\begin{array}{l}\text { alignment at the air/LC } \\
\text { interface (at } 24^{\circ} \mathrm{C} \text { ) }\end{array}$ & $\begin{array}{l}\text { alignment at the OTS-glass/LC } \\
\text { interface (at } 24{ }^{\circ} \mathrm{C} \text { ) }\end{array}$ & $\begin{array}{c}\text { surface tension }\left(\mathrm{N} \mathrm{m}^{-1} \times\right. \\
\left.10^{-3}\right)\left(\text { at } 24{ }^{\circ} \mathrm{C}\right)\end{array}$ \\
\hline MLC6609 & 91.5 & 15.3 & 15.9 & 1.04 & -3.3 & homeotropic & homeotropic & 23.4 \\
\hline E7 & 60.5 & $11.1^{d}$ & $17.1^{d}$ & $1.54^{d}$ & $+14.1^{d}$ & homeotropic & homeotropic & 24.2 \\
\hline $6 \mathrm{CB}$ & 29.0 & $5.4^{e}$ & $6.4^{e}$ & 1.19 & $+11.2^{e}$ & homeotropic & homeotropic & 31.1 \\
\hline $5 \mathrm{CB}$ & 35.5 & $7.4^{e}$ & $10.5^{e}$ & 1.42 & $+14.6^{e}$ & homeotropic & homeotropic & 32.8 \\
\hline MLC2081 & 106.0 & 14.2 & 20.4 & 1.44 & -3.8 & planar & planar & 24.2 \\
\hline MLC7023 & 79.5 & 11.1 & 13.3 & 1.20 & +6.6 & planar & planar & low $^{c}$ \\
\hline
\end{tabular}

${ }^{a}$ For ease of comparison, the data in this table are displayed with the LCs in the same order as in Table 1. Data taken from the literature are indicated. ${ }^{b}$ From ref $49 .{ }^{c}$ Too low to be measured by the pendant drop method. ${ }^{d}$ Merck data sheets $\left(K_{11}, K_{33}\right.$, and $\Delta \varepsilon$ measured at $\left.20{ }^{\circ} \mathrm{C}\right) .{ }^{e}$ From ref $50\left(K_{11}, K_{33}\right.$, and $\Delta \varepsilon$ measured at $\left.20^{\circ} \mathrm{C}\right)$.

normal "bright-field" microscopy (Figure 6a,c,e). In cases where the alignment at the air interface is homeotropic
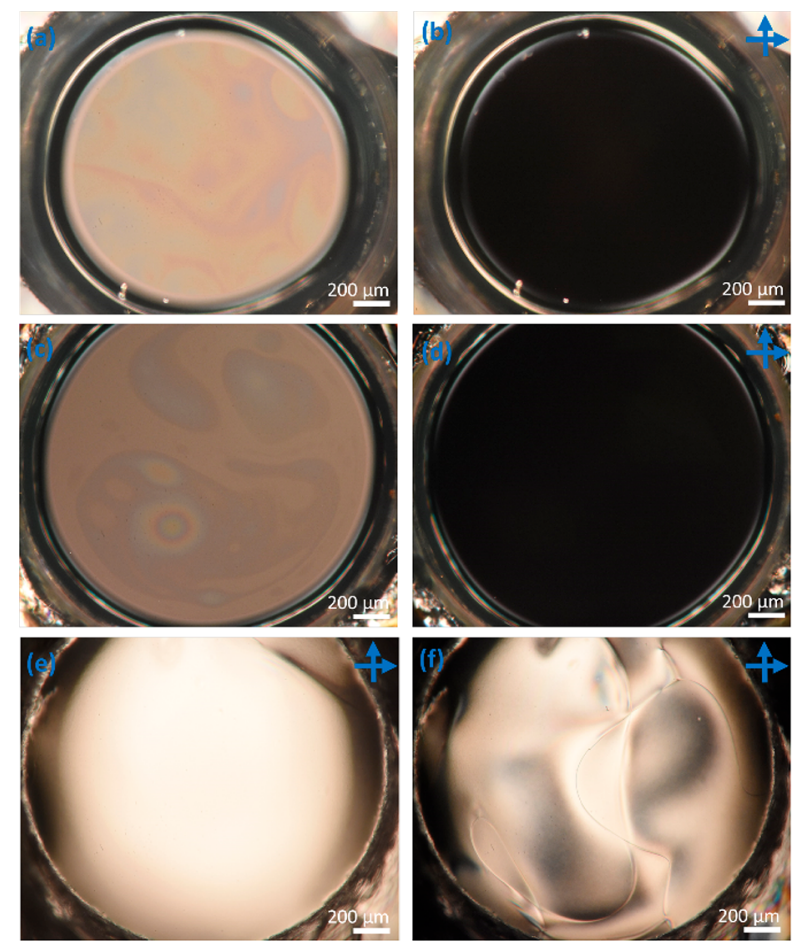

Figure 6. Transmission POM images of freely suspended LC films at an air interface. (a) Bright-field image of a 5CB film. (b) Image between crossed polarizers of a 5CB film showing homeotropic (perpendicular) alignment at an air interface. (c) Bright-field image of an E7 film. (d) Image between crossed polarizers of an E7 film showing homeotropic (perpendicular) alignment at an air interface. (e) Bright-field image of a MLC7023 film. (f) Image between crossed polarizers of a MLC7023 film, showing nonhomeotropic alignment at an air interface.

(perpendicular), the expected black field was observed (Figure $6 \mathrm{~b}, \mathrm{~d})$, except around the edges of the hole where a bright rim, due to misalignment on the rough edges of the hole, is apparent. In cases where the alignment is nonhomeotropic a bright birefringent "texture" is observed (Figure 6f). It is not possible to conclude from these experiments whether this birefringence is due to a tilted degenerate or planar degenerate alignment at the air interface.

The alignment of these LCs was also investigated at the OTS-coated glass interface. In cases where the director aligns in a homeotropic (perpendicular) manner, the use of crossed polarizers produces a black image except at edge of the sample where a birefringent border is observed, as seen in Figure 7a; for example, in cases where alignment is nonhomeotropic, the normal Schlieren texture of the nematic phase is observed (Figure 7b).
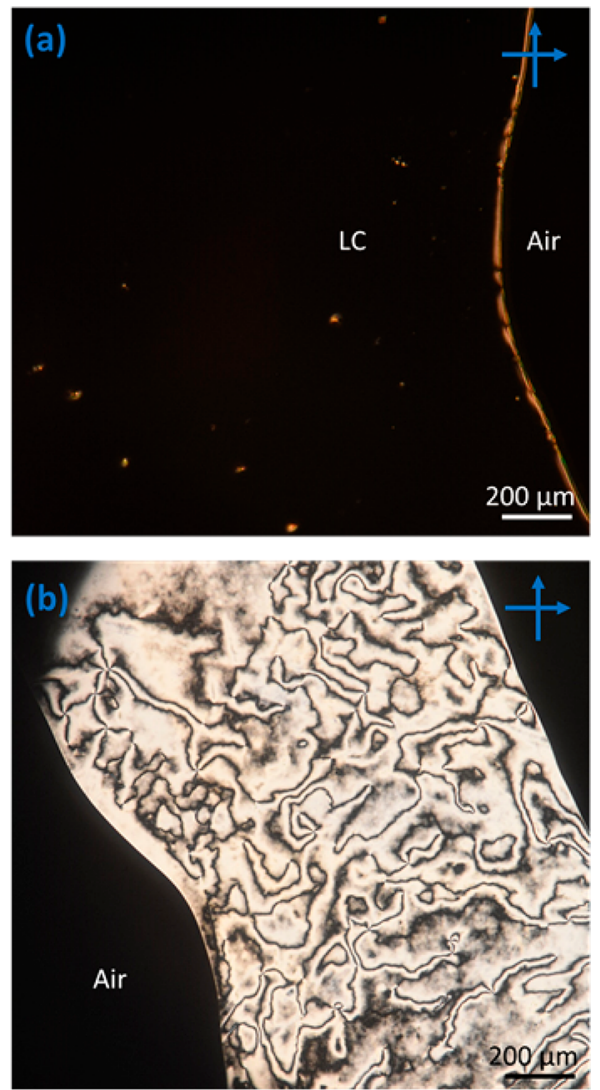

Figure 7. Transmission POM images of LCs confined between OTScoated glass substrates: (a) 6CB and (b) MLC7023.

We see a direct a correlation between the observed alignment for these LCs materials at the air interface and their alignment on OTS-coated glass. More importantly, there is a broad correlation between their alignment at the air or OTS surfaces (Table 3) and the observed director fields in the phospholipid-coated LC droplets (Table 1); the director fields observed in the droplets correlate with the intrinsic LC surface rather than the intrinsic LC bulk properties. For these LC materials that align in a homeotropic manner at the air interface and/or on OTS glass, in 14 of the 16 types of lipidcoated droplets the director field is radial; the alignment in the droplet is also homeotropic. The exceptions are those cases 
(6CB and 5CB) where the lipid is DFPC-the lipid least favorable to homeotropic anchoring. For the two LCs that align in a nonhomeotropic manner at the air interface and on OTS glass in six/seven of the eight types of lipid-coated droplets the director field is bipolar: the alignment in the droplet is also nonhomeotropic. The only clear exception is for MLC2081 coated with DOPC:DOPG-the lipid mixture most favorable to homeotropic anchoring.

To understand this dependence on the surface properties of the LCs in more detail, we measured their surface tensions at the LC/air interface. This was done by the pendent drop method. $^{32,33}$ For MLC7023 were we unable to attain a consistent "plateau region" value for the surface tension/ droplet volume plots. The droplet fell off the needle before reaching a volume of $4 \mu \mathrm{L}$, and no "plateau region" was obtained. However, this fact together with the slope of the "apparent surface tension"/volume plot compared to that for the other systems suggested a very low surface tension. (This mixture is designed for operation in TFT displays and includes a high proportion of highly fluorinated end groups.) A comparison of surface tensions obtained for $5 \mathrm{CB}$ and $6 \mathrm{CB}$ (Table 3) with literature values ${ }^{49}$ shows quite good agreement.

Figure 8 shows examples of the drop shapes for the LCs studied at the point just before they dropped from the needle. In a qualitative sense it can be seen that the LCs with the lower surface tension produce a more elongated droplet shape whereas the materials with higher surface tension produce a more rounded droplet. It further supports the idea that the surface tension for MLC7023 is very low (Figure S34). As can be seen from the surface tension values for the first four entries in Table 3, the entries for the LCs that align in a perpendicular manner at the air and OTS interfaces (5CB and 6CB) are those associated with the higher surface tension values. It is perhaps significant to note that it is also these LCs that with the phospholipid least favorable to perpendicular alignment, DFPC, give the nonradial droplets (Table 1). There is insufficient experimental data (and in particular we were unable to obtain surface tension data) to be able to understand the factors involved in the radial nature of the MLC2081 droplets coated with DOPC:DOPG.

\section{CONCLUSIONS}

Sometimes the alignment of LCs at interfaces is discussed as if it were determined solely by the interaction forces between the LC molecules and the surface. This is not the case: the bulk mechanical properties of the LC are also important. ${ }^{47}$ For example, if the attractive interactions between the molecules within the LC phase ${ }^{51}$ are very strong and they are highly anisotropic (an intrinsic bulk property of the LC), this alone determines the surface alignment. ${ }^{52}$ Another very important factor is the entropic cost associated the local smectic ordering required for perpendicular anchoring. For the simple alkyl cyanobiphenyls the ability of the system to achieve this local smectic order increases with increasing end-chain length and follows the order $5 \mathrm{CB}>6 \mathrm{CB}>7 \mathrm{CB}>8 \mathrm{CB} .{ }^{47,53,54}$ For $5 \mathrm{CB}$ and $6 \mathrm{CB}$ this is also the order seen for the surface tension values for the perpendicular (homeotropically) ordered air/LC interface (Table 3).

Table 1 shows that the director fields for the phospholipidcoated LC droplets are dependent on both the nature of the phospholipid and that of the LC. The data are arranged in terms of the ability of the two components to promote either radial (including preradial/escaped radial) or nonradial (axial
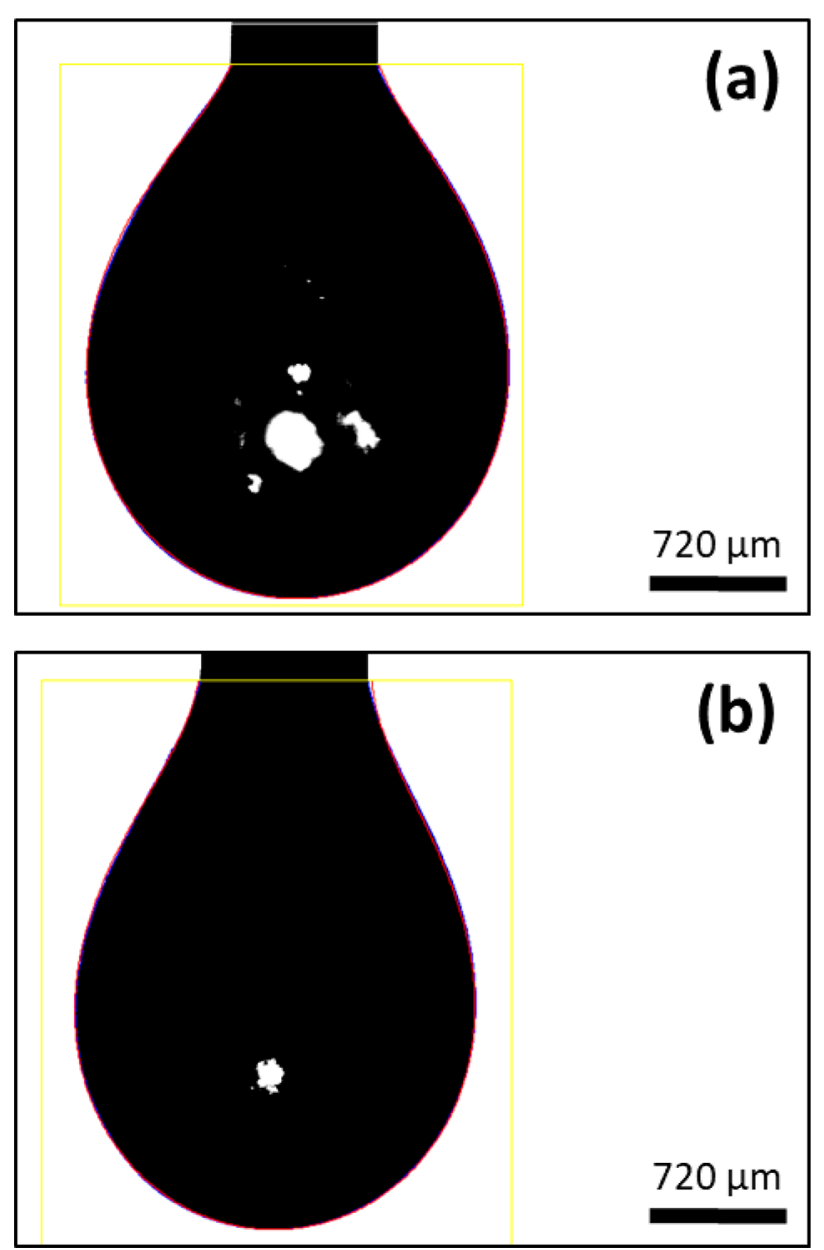

Figure 8. Pendant drop images of LCs observed at $22{ }^{\circ} \mathrm{C}$ : (a) $5 \mathrm{CB}$ and (b) MLC2081. The difference in shape suggests a clear difference in surface tension of the LCs in air. The red line following the contour of the droplet represents the calculated fit to the Young-Laplace equation.

and bipolar) ordering. In the resultant "phase space" the order in which the phospholipid components need to be arranged is that qualitatively expected in terms of ease of interpenetration of the LC molecules into a fluid "normal" phospholipid monolayer. This is probably not the only factor involved, ${ }^{45,46}$ but it seems to be the major influence. The order seen for the LC component is less easy to understand, but our studies suggest that for these droplets, at least, the intrinsic surface rather than the intrinsic bulk properties is the most important. This is perhaps surprising since, if surface anchoring, $\mathrm{W}$ is not very strong, the alignment of LCs in droplets has so often been discussed in terms of the Frank elastic constants ${ }^{55,56}$ (an intrinsic bulk property). For some droplet systems this is doubtlessly important; ${ }^{17}$ there is some evidence for this in the case of MLC2081 and MLC7023, but for these phospholipidcoated LC droplets comparison of the observed director fields with the measured values of $K_{11}, K_{33}$, and $K_{33} / K_{11}$, shows no correlation with the observed experimental behavior as there is very little variation in the $K_{33} / K_{11}$ ratio. It would typically be expected that for droplets with a low W a $K_{33} / K_{11}>1$ would promote bipolar anchoring and a $K_{33} / K_{11}<1$ would suggest a nonplanar anchoring. Introduction of a small amount of twist into the director field models for the radial and bipolar droplets yields a more complex picture in which $K_{22}$ (the twist elastic 
constant) also plays a role. Whether knowledge of these values for the LCs we have used or inclusion of a contribution from the saddle-splay elastic constant $K_{24}$ would modify this conclusion is not clear. It has been reported recently by Abbott that by utilizing a "one elastic constant approximation" (i.e., $K=K_{11}=K_{22}=K_{33}$ ) and incorporating the contribution from $K_{24}$ to the Frank-Oseen equation it is possible to predict the droplet size at which a uniform radial configuration is stabilized. ${ }^{57}$ The free energy of radial configuration $\left(F_{\mathrm{R}}\right)$ can be described by

$$
F_{\mathrm{R}}=8 \pi K_{11} R-4 \pi K_{24} R+2 \pi W R^{2}
$$

where the first term is associated with the splay deformation within the droplet, the second term the saddle-splay deformation, which favors a radial droplet alignment, and the third term the energetic penalty associated with a homeotropic alignment at the droplet interface. While it has been demonstrated this can be used to predict droplet alignment, there remain issues; for example, experimental estimates of $K_{24}$ vary significantly. Our studies of free-standing films, where $K_{24}$ is not expected to be significant, give some insight into whether the saddle-splay constant is important in this study. We see a strong correlation between the film and droplet geometries for the different liquid crystals considered, suggesting that the $K_{24}$ term in eq 1 is not dominant in our system. It would be interesting to undertake detailed experiments in the future, though as mentioned, measurements of $K_{24}$ are extremely challenging.

Overall, the data strongly suggest that for these droplets, at least, the intrinsic surface and not intrinsic bulk LC properties is the dominant factor. What does correlate in an obvious manner with the experimentally observed director fields is the alignment behavior of these LCs at the air (and at the OTStreated glass) interface (Table 3 ). Those LCs where the director field is homeotropic (perpendicular) at the air interface give mainly radial director fields in the lipid-coated droplets, and those which are planar at the air interface give mainly nonradial director fields. Within these two broad categories the energy required to form a particular type of interfacial layer (be it perpendicular or planar) is measured by the surface tension. For the perpendicular/radial director field it is those systems with a low surface tension which give a radial field for all four lipid systems. It is the "single component" LC systems, 5CB and 6CB, for which the "energy cost" of forming a homeotropic surface (the surface tension) is highest that for the DFPC give a bipolar director field.

Phospholipid-coated LC droplets are particularly attractive candidates for forming biosensors. The ambipolar nature of the lipid monolayer provides a natural "bridge" between the hydrophobic LC and the water-based world of biology. Furthermore, the large number of sensor molecules that exist within biomembranes can potentially be exploited. In terms of this potential use of phospholipid-coated LC droplets as sensor systems several of the results described in this study are of importance. First, it has been shown that by using synthetic lipids with bulky acyl group end-groups phospholipid coatings can be used to promote either perpendicular or planar anchoring. This means that it is possible to envisage sensor systems in which switching is either radial to bipolar or bipolar to radial. Second, it has been shown that this alignment depends on not only the choice of lipid but also the choice of LC. For example, with MLC7023, it is possible to achieve planar anchoring even with a "conventional" phospholipid mixture. Third, it has been shown that the director field (in droplets of this particular type) is determined by the intrinsic surface properties rather than the bulk elastic properties of the LC. Hence, they should be responsive to changes in surface anchoring energy. Fourth, ongoing work (to be published) has shown that we can control not only the sign of the surface anchoring but also (at least potentially) its strength by using a mixture of lipids and a mixture of LCs. This is likely to prove important in controlling the sensitivity of any sensor device produced.

\section{ASSOCIATED CONTENT}

\section{Supporting Information}

The Supporting Information is available free of charge at https://pubs.acs.org/doi/10.1021/acs.langmuir.0c00651.

Details of materials and instrumentation, full synthetic details for the lipids DnPdPC, MFPC, and DFPC, DSC data for the synthetic lipids MFPC and DFPC, collapse pressure data from Langmuir trough experiments, POM and fluorescent images of lipid-coated LC droplets and pendant drop images (PDF)

\section{AUTHOR INFORMATION}

\section{Corresponding Author}

Richard J. Bushby - School of Chemistry, University of Leeds, Leeds LS2 9JT, U.K.; 이이이.org/0000-0002-1627-6058; Email: r.j.bushby@leeds.ac.uk

\section{Authors}

Daniel A. Paterson - School of Physics and Astronomy and School of Chemistry, University of Leeds, Leeds LS2 9JT, U.K.; (1) orcid.org/0000-0001-9425-720X

Peng Bao - School of Physics and Astronomy, University of

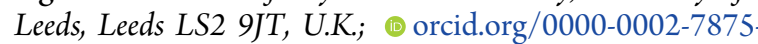
7420

Radwa H. Abou-Saleh - School of Physics and Astronomy, University of Leeds, Leeds LS2 9JT, U.K.; Biophysics Group, Department of Physics, Faculty of Science, Mansoura University, Mansoura, Egypt; 이이이.org/0000-0002-8471-2659

Sally A. Peyman - School of Physics and Astronomy and School of Medicine, University of Leeds, Leeds LS2 9JT, U.K.

J. Cliff Jones - School of Physics and Astronomy, University of Leeds, Leeds LS2 9JT, U.K.; (1) orcid.org/0000-0002-23100800

Jonathan A. T. Sandoe - Leeds Institute of Medical Research, University of Leeds, Leeds LS2 9JT, U.K.; 이이.org/00000003-0193-8677

Stephen D. Evans - School of Physics and Astronomy, University of Leeds, Leeds LS2 9JT, U.K.; 이이.org/00000001-8342-5335

Helen F. Gleeson - School of Physics and Astronomy, University of Leeds, Leeds LS2 9JT, U.K.; 이이.org/00000002-7494-2100

Complete contact information is available at:

https://pubs.acs.org/10.1021/acs.langmuir.0c00651

\section{Author Contributions}

Most of the experimental work was performed by D.A.P., but the lipid characterization was mainly due to P.B. and R.H.A.-S. The overall project coordinators are H.F.G., S.D.E., and R.J.B. All authors contributed to the writing of the manuscript. 


\section{Notes}

The authors declare no competing financial interest.

\section{ACKNOWLEDGMENTS}

The authors thank Merck Group, UK, for providing materials MLC6609, MLC2081, and MLC7023. The authors gratefully acknowledge the financial support from EPSRC (UK) with Grant EP/P024041/1. S.D.E. is also supported by Health Services and Delivery Research Programme, Grant/Award MIC-2016-004 and MRC: MR/M009084/1. R.J.B., S.D.E., R.H.A.-S., and S.A.P. also acknowledge support from the EPSRC EP/EP/P023266/1. J.C.J. acknowledges support from EPSRC for a Fellowship in Manufacturing (EP/L015188/2). S. Srigengan is thanked for his help with the Freedericksz transition measurements.

\section{REFERENCES}

(1) Cronin, T. Biosensors and Liquid Crystals. In Handbook of Liquid Crystals; Goodby, J. W., Tschierske, C., Raynes, P., Gleeson, H., Kato, T., Collings, P. J., Eds.; Wiley-VCH Verlag GmbH \& Co., 2014. DOI: $10.1002 / 9783527671403 . h l c 156$.

(2) Woltman, S. J.; Jay, G. D.; Crawford, G. P. Liquid-crystal materials find a new order in biomedical applications. Nat. Mater. 2007, 6, 929.

(3) Carlton, R. J.; Hunter, J. T.; Miller, D. S.; Abbasi, R.; Mushenheim, P. C.; Tan, L. N.; Abbott, N. L. Chemical and biological sensing using liquid crystals. Liq. Cryst. Rev. 2013, 1 (1), 29-51.

(4) Lowe, A. M.; Abbott, N. L. Liquid Crystalline Materials for Biological Applications. Chem. Mater. 2012, 24 (5), 746-758.

(5) Popov, P.; Mann, E. K.; Jakli, A. Thermotropic liquid crystal films for biosensors and beyond. J. Mater. Chem. B 2017, 5 (26), 5061-5078.

(6) Setia, S.; Sidiq, S.; De, J.; Pani, I.; Pal, S. K. Applications of liquid crystals in biosensing and organic light-emitting devices: future aspects. Liq. Cryst. 2016, 43 (13-15), 2009-2050.

(7) Popov, P.; Honaker, L. W.; Kooijman, E. E.; Mann, E. K.; Jákli, A. I. A liquid crystal biosensor for specific detection of antigens. Sensing and Bio-Sensing Research 2016, 8, 31-35.

(8) Khan, M.; Khan, A. R.; Shin, J.-H.; Park, S.-Y. A liquid-crystalbased DNA biosensor for pathogen detection. Sci. Rep. 2016, 6, 22676.

(9) Brake, J. M.; Daschner, M. K.; Luk, Y.-Y.; Abbott, N. L. Biomolecular Interactions at Phospholipid-Decorated Surfaces of Liquid Crystals. Science 2003, 302 (5653), 2094.

(10) Manna, U.; Zayas-Gonzalez, Y. M.; Carlton, R. J.; Caruso, F.; Abbott, N. L.; Lynn, D. M. Liquid Crystal Chemical Sensors That Cells Can Wear. Angew. Chem., Int. Ed. 2013, 52 (52), 14011-14015.

(11) Sidiq, S.; Prasad, G. V. R. K.; Mukhopadhaya, A.; Pal, S. K. Poly(1-lysine)-Coated Liquid Crystal Droplets for Cell-Based Sensing Applications. J. Phys. Chem. B 2017, 121 (16), 4247-4256.

(12) Lee, H.-G.; Munir, S.; Park, S.-Y. Cholesteric Liquid Crystal Droplets for Biosensors. ACS Appl. Mater. Interfaces 2016, 8 (39), 26407-26417.

(13) Deng, J.; Liang, W.; Rhodes, S.; Fang, J. Influence of polymer networks on the sensor properties of hydrogel dispersed liquid crystal droplets. Colloids Surf., A 2019, 570, 438-443.

(14) Sidiq, S.; Das, D.; Pal, S. K. A new pathway for the formation of radial nematic droplets within a lipid-laden aqueous-liquid crystal interface. RSC Adv. 2014, 4 (36), 18889-18893.

(15) Lowe, A. M.; Ozer, B. H.; Bai, Y.; Bertics, P. J.; Abbott, N. L. Design of Surfaces for Liquid Crystal-Based Bioanalytical Assays. ACS Appl. Mater. Interfaces 2010, 2 (3), 722-731.

(16) Bao, P.; Paterson, D. A.; Harrison, P. L.; Miller, K.; Peyman, S.; Jones, J. C.; Sandoe, J.; Evans, S. D.; Bushby, R. J.; Gleeson, H. F. Lipid coated liquid crystal droplets for the on-chip detection of antimicrobial peptides. Lab Chip 2019, 19, 1082.
(17) Miller, D. S.; Abbott, N. L. Influence of droplet size, $\mathrm{pH}$ and ionic strength on endotoxin-triggered ordering transitions in liquid crystalline droplets. Soft Matter 2013, 9 (2), 374-382.

(18) He, S.; Liang, W.; Cheng, K.-L.; Fang, J.; Wu, S.-T. Bile acidsurfactant interactions at the liquid crystal/aqueous interface. Soft Matter 2014, 10 (26), 4609-4614.

(19) Gupta, J. K.; Sivakumar, S.; Caruso, F.; Abbott, N. L. SizeDependent Ordering of Liquid Crystals Observed in Polymeric Capsules with Micrometer and Smaller Diameters. Angew. Chem., Int. Ed. 2009, 48 (9), 1652-1655.

(20) Lavrentovich, O. D. Topological defects in dispersed words and worlds around liquid crystals, or liquid crystal drops. Liq. Cryst. 1998, 24 (1), 117-126.

(21) Wang, X.; Zhou, Y.; Kim, Y.-K.; Miller, D. S.; Zhang, R.; Martinez-Gonzalez, J. A.; Bukusoglu, E.; Zhang, B.; Brown, T. M.; de Pablo, J. J.; Abbott, N. L. Patterned surface anchoring of nematic droplets at miscible liquid-liquid interfaces. Soft Matter 2017, 13 (34), 5714-5723.

(22) Brake, J. M.; Mezera, A. D.; Abbott, N. L. Effect of Surfactant Structure on the Orientation of Liquid Crystals at Aqueous-Liquid Crystal Interfaces. Langmuir 2003, 19 (16), 6436-6442.

(23) Lockwood, N. A.; de Pablo, J. J.; Abbott, N. L. Influence of Surfactant Tail Branching and Organization on the Orientation of Liquid Crystals at Aqueous-Liquid Crystal Interfaces. Langmuir 2005, 21 (15), 6805-6814.

(24) Brake, J. M.; Mezera, A. D.; Abbott, N. L. Active Control of the Anchoring of 4-Pentyl-4-cyanobiphenyl (5CB) at an AqueousLiquid Crystal Interface By Using a Redox-Active Ferrocenyl Surfactant. Langmuir 2003, 19 (21), 8629-8637.

(25) Bao, P.; Cheetham, M. R.; Roth, J. S.; Blakeston, A. C.; Bushby, R. J.; Evans, S. D. On-chip alternating current electrophoresis in supported lipid bilayer membranes. Anal. Chem. 2012, 84 (24), 10702-10707.

(26) Wang, X.; Bukusoglu, E.; Abbott, N. L. A Practical Guide to the Preparation of Liquid Crystal-Templated Microparticles. Chem. Mater. 2017, 29 (1), 53-61.

(27) Abou-Saleh, R. H.; McLaughlan, J. R.; Bushby, R. J.; Johnson, B. R.; Freear, S.; Evans, S. D.; Thomson, N. H. Molecular Effects of Glycerol on Lipid Monolayers at the Gas-Liquid Interface: Impact on Microbubble Physical and Mechanical Properties. Langmuir 2019, 35 (31), 10097-10105.

(28) Emile, J.; Casanova, F.; Loas, G.; Emile, O. Swelling of a foam lamella in a confined channel. Soft Matter 2012, 8 (27), 7223-7227.

(29) Axelrod, D.; Koppel, D. E.; Schlessinger, J.; Elson, E.; Webb, W. W. Mobility measurement by analysis of fluorescence photobleaching recovery kinetics. Biophys. J. 1976, 16 (9), 1055-1069.

(30) Srigengan, S.; Nagaraj, M.; Ferrarini, A.; Mandle, R.; Cowling, S. J.; Osipov, M. A.; Pajạk, G.; Goodby, J. W.; Gleeson, H. F. Anomalously low twist and bend elastic constants in an oxadiazolebased bent-core nematic liquid crystal and its mixtures; contributions of spontaneous chirality and polarity. J. Mater. Chem. C 2018, 6 (5), 980-988.

(31) Kaur, S.; Liu, H.; Addis, J.; Greco, C.; Ferrarini, A.; Görtz, V.; Goodby, J. W.; Gleeson, H. F. The influence of structure on the elastic, optical and dielectric properties of nematic phases formed from bent-core molecules. J. Mater. Chem. C 2013, 1 (40), 66676676.

(32) Carboni, C.; Al-Ruzaiqi, S.; Al-Siyabi, A.; Al-Harthi, S.; George, A. K.; Sluckin, T. J. The Behaviour of the Interfacial Surface Tension of Liquid-Crystal Materials in the Vicinity of the Nematic-Isotropic Phase Transition. Mol. Cryst. Liq. Cryst. 2008, 494 (1), 114-126.

(33) Daerr, A. A. M. A. Pendent Drop: An ImageJ Plugin to Measure the Surface Tension from an Image of a Pendent Drop. J. Open Res. Software [Online] 2016, p.e3.

(34) Guo, J.-K.; Hong, S.-H.; Yoon, H.-J.; Babakhanova, G.; Lavrentovich, O. D.; Song, J.-K. Laser-Induced Nanodroplet Injection and Reconfigurable Double Emulsions with Designed Inner Structures. Advanced Science 2019, 6 (17), 1900785. 
(35) Solodkov, N. V.; Shim, J.-u.; Jones, J. C. Self-assembly of fractal liquid crystal colloids. Nat. Commun. 2019, 10 (1), 198.

(36) Lin, I. H.; Miller, D. S.; Bertics, P. J.; Murphy, C. J.; de Pablo, J. J.; Abbott, N. L. Endotoxin-Induced Structural Transformations in Liquid Crystalline Droplets. Science 2011, 332 (6035), 1297.

(37) Brake, J. M.; Daschner, M. K.; Abbott, N. L. Formation and Characterization of Phospholipid Monolayers Spontaneously Assembled at Interfaces between Aqueous Phases and Thermotropic Liquid Crystals. Langmuir 2005, 21 (6), 2218-2228.

(38) Wang, X.; Miller, D. S.; Bukusoglu, E.; de Pablo, J. J.; Abbott, N. L. Topological defects in liquid crystals as templates for molecular self-assembly. Nat. Mater. 2016, 15 (1), 106-112.

(39) Wang, X.; Kim, Y.-K.; Bukusoglu, E.; Zhang, B.; Miller, D. S.; Abbott, N. L. Experimental Insights into the Nanostructure of the Cores of Topological Defects in Liquid Crystals. Phys. Rev. Lett. 2016, 116 (14), 147801.

(40) Sobotka, H.; Rosenberg, S.; Birnbaum, A. Films of OmegaBranched Fatty Acids. J. Colloid Sci. 1950, 5 (6), 567-580.

(41) Kiselev, V. D.; Potapova, L. N.; Kashaeva, H. A.; Konovalov, A. I. Solvent effect on the partial molar volume and solution enthalpy of ferrocene. Mendeleev Commun. 2012, 22 (1), 50-51.

(42) Fuks, S.; Legros, J. C.; Bellemans, A. The molar volumes of liquid methane and deuteromethane. Physica 1965, 31 (4), 606-612.

(43) Ma, G.; Allen, H. C. DPPC Langmuir Monolayer at the AirWater Interface: Probing the Tail and Head Groups by Vibrational Sum Frequency Generation Spectroscopy. Langmuir 2006, 22 (12), 5341-5349.

(44) Blume, A. Lipids at the air-water interface. ChemTexts 2018, 4 (1), 3.

(45) Gallardo, B. S.; Metcalfe, K. L.; Abbott, N. L. Ferrocenyl Surfactants at the Surface of Water: Principles for Active Control of Interfacial Properties. Langmuir 1996, 12 (17), 4116-4124.

(46) Kim, Y.-K.; Huang, Y.; Tsuei, M.; Wang, X.; Gianneschi, N. C.; Abbott, N. L. Multi-Scale Responses of Liquid Crystals Triggered by Interfacial Assemblies of Cleavable Homopolymers. ChemPhysChem 2018, 19 (16), 2037-2045.

(47) Alkhairalla, B.; Allinson, H.; Boden, N.; Evans, S. D.; Henderson, J. R. Anchoring and orientational wetting of nematic liquid crystals on self-assembled monolayer substrates: An evanescent wave ellipsometric study. Phys. Rev. E: Stat. Phys., Plasmas, Fluids, Relat. Interdiscip. Top. 1999, 59 (3), 3033-3039.

(48) Kralj, S.; Žumer, S. Freedericksz transitions in supra- $\mu \mathrm{m}$ nematic droplets. Phys. Rev. A: At., Mol., Opt. Phys. 1992, 45 (4), 2461-2470.

(49) Gomes, L. S.; Demarquette, N. R. Influence of Temperature on Surface Tension of Liquid Crystals in the Cyanobiphenyl and Cyanooxybiphenyl Series. Mol. Cryst. Liq. Cryst. 2005, 437 (1), 181-194.

(50) Bradshaw, M. J.; Raynes, E. P.; Bunning, J. D.; Faber, T. E. The Frank constants of some nematic liquid crystals. J. Phys. (Paris) 1985, 46 (9), 1513-1520.

(51) Jones, S. A.; Bailey, J.; Walker, D. R. E.; Bryan-Brown, G. P.; Jones, J. C. Method for Tuneable Homeotropic Anchoring at Microstructures in Liquid Crystal Devices. Langmuir 2018, 34 (37), $10865-10873$.

(52) Wasserfallen, D.; Kastler, M.; Pisula, W.; Hofer, W. A.; Fogel, Y.; Wang, Z.; Müllen, K. Suppressing Aggregation in a Large Polycyclic Aromatic Hydrocarbon. J. Am. Chem. Soc. 2006, 128 (4), 1334-1339.

(53) Sadati, M.; Ramezani-Dakhel, H.; Bu, W.; Sevgen, E.; Liang, Z.; Erol, C.; Rahimi, M.; Taheri Qazvini, N.; Lin, B.; Abbott, N. L.; Roux, B.; Schlossman, M. L.; de Pablo, J. J. Molecular Structure of Canonical Liquid Crystal Interfaces. J. Am. Chem. Soc. 2017, 139 (10), 38413850.

(54) Ramezani-Dakhel, H.; Sadati, M.; Rahimi, M.; RamirezHernandez, A.; Roux, B.; de Pablo, J. J. Understanding AtomicScale Behavior of Liquid Crystals at Aqueous Interfaces. J. Chem. Theory Comput. 2017, 13 (1), 237-244.

(55) Lopez-Leon, T.; Fernandez-Nieves, A. Drops and shells of liquid crystal. Colloid Polym. Sci. 2011, 289 (4), 345-359.
(56) Tomar, V.; Hernández, S. I.; Abbott, N. L.; Hernández-Ortiz, J. P.; de Pablo, J. J. Morphological transitions in liquid crystal nanodroplets. Soft Matter 2012, 8 (33), 8679-8689.

(57) Miller, D. S.; Wang, X.; Abbott, N. L. Design of Functional Materials Based on Liquid Crystalline Droplets. Chem. Mater. 2014, 26 (1), 496-506. 